\title{
Thermoluminescence Glow Curve Analysis of Thermoluminescent Na-Doped BeO Phosphor Using a Postannealing Method with Very Low Heating Rate
}

\author{
Kiyomitsu Shinsho, ${ }^{*}$ Keisuke Otsubo, Yusuke Koba, ${ }^{1}$ \\ Kazuki Matsumoto, ${ }^{2}$ and Hiroaki Ushiba ${ }^{2}$ \\ Division of Radiological Sciences, Faculty of Health Sciences, Tokyo Metropolitan University, \\ 7-2-10 Higashi-Ogu, Arakawa-ku, Tokyo 116-8551, Japan \\ ${ }^{1}$ Medical Exposure Research Project, National Institute of Radiological Sciences, \\ 4-9-1 Anagawa, Inage-ku, Chiba 263-8555, Japan \\ ${ }^{2}$ Chiyoda Technol Corporation, 2-16-2 Sotokanda, Chiyoda-ku, Tokyo 101-0021, Japan
}

(Received February 3, 2016; accepted May 19, 2016)

Keywords: thermoluminescence, TL glow curve, $\mathrm{BeO}$, radiation dosimetry

The thermoluminescent Na-doped $\mathrm{BeO}$ phosphor (Panasonic UD-170A) is one of the better thermoluminescent dosimeters due to its high sensitivity, tissue equivalency, and repeatability. However, there have only been a few reports on thermoluminescence (TL) glow component analysis, despite this being necessary to understand the physical characteristics of thermoluminescent phosphors based on $\mathrm{BeO}$. In this study, the TL glow curve of the thermoluminescent $\mathrm{Na}$-doped $\mathrm{BeO}$ phosphor was analyzed using a postannealing method with a very low heating rate of $0.1{ }^{\circ} \mathrm{C} \mathrm{s}^{-1}$. Although the TL glow curve for thermoluminescent Na-doped $\mathrm{BeO}$ phosphor was previously reported to consist of only a few components, our investigation analyzed at least thirteen components in the TL glow curve. In addition, the accuracy of the TL parameters calculated in this study was very high using a postannealing method with a very low heating rate.

\section{Introduction}

Tochilin et al. first reported $\mathrm{BeO}$ as a thermoluminescent dosimeter in 1969.(1) Thermoluminescent phosphors based on $\mathrm{BeO}$ were then commercialized in 1975 by Yasuno et al., (2) due to their good thermoluminescence (TL) properties with high sensitivity, repeatability, low fading, tissue equivalency $\left(Z_{\text {eff }}=7.1\right)$, and low neutron sensitivity. (3) Since then, there have been many reports on the properties and applications of thermoluminescent phosphors based on $\mathrm{BeO} .{ }^{(4-8)}$ However, there have been only a few reports on TL glow component analysis, despite this being necessary to understand the physical characteristics of TL based on BeO. Nieto et al. reported studies on the kinetic parameters of undoped $\mathrm{BeO}$ using an isothermal decay method, for which the calculated activation energy $(E)$ was in agreement with that obtained using the TL glow curve shape with Chen's modified method; however, the frequency factor $(s)$ differed considerably due to the inclusion of an experimental error.(7) Finally, Nieto et al. mentioned that as $E$ enters are an exponent in the expression for $s$, a small variation in $E$ leads to significant changes in the calculated values of ${ }^{*}$ Corresponding author: e-mail: shinsho@tmu.ac.jp 
$s$. Therefore, the most important thing in determining the kinetic parameters is to measure highly precise TL glow curves. We suggest that TL glow curve measurement at a very low heating rate would be an effective means to resolve this issue because the temperature of the thermoluminescent phosphor can be precisely controlled.

Therefore, in this study, the TL glow curve of the thermoluminescent Na-doped BeO phosphor was analyzed using a postannealing method with a very low heating rate of $0.1^{\circ} \mathrm{C} \mathrm{s}^{-1}$.

\section{Materials and Methods}

\subsection{TL phosphor}

Na-doped $\mathrm{BeO}$ (Panasonic UD-170A) was used as the thermoluminescent phosphor. The diameter was $2 \mathrm{~mm}$ and the length was $12 \mathrm{~mm}$.

\subsection{TL glow curve measurements}

$\mathrm{X}$-ray irradiation was carried out using a Varian CLINAC-21EX linear accelerator at $6 \mathrm{MV}$. The TL samples and a Scanditronix CC13 ion chamber were also positioned in a tough water-equivalent slab phantom at a depth of $10 \mathrm{~cm}$. The source-to-chamber distance was $100 \mathrm{~cm}$ and the dose was 5 Gy. Figure 1 shows a photograph of the TL glow curve measurement system used in this work. TL glow curves were recorded from room temperature up to $350{ }^{\circ} \mathrm{C}$ at a heating rate of $0.1{ }^{\circ} \mathrm{C} \mathrm{s}^{-1}$ in air. The data were acquired at $10 \mathrm{~s}$ intervals with a sampling time of $50 \mathrm{~m}$. The temperature was controlled using a programmable thermoregulator (Sakaguchi E.H VOC Corp. SCR-SHQ-A). A photon-counting head light detector (Hamamatsu H11890-210) was used with a spectral sensitivity region of 400-700 nm. No spectroscopic attachments were used aside from a thermal-cut filter. The postannealing temperatures for irradiated samples were in the range of $90-200{ }^{\circ} \mathrm{C}$ at $10{ }^{\circ} \mathrm{C}$ intervals. The postannealing duration was $60 \mathrm{~m}$.

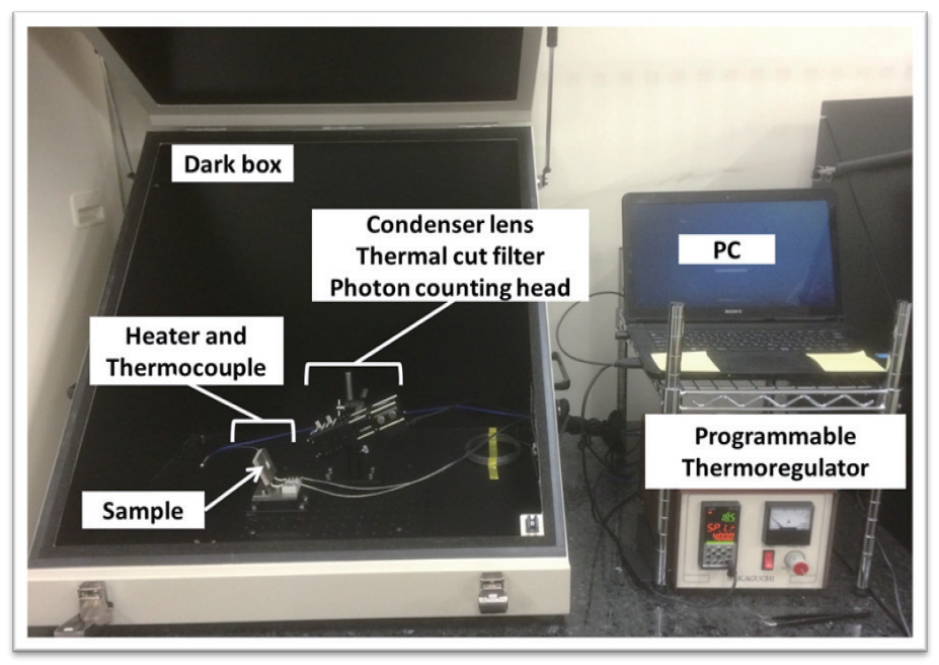

Fig. 1. (Color online) TL glow curve measurement system. 
The TL spectroscopic analysis was performed using a HAMAMATSU PMA-11 multichannel charge-coupled device (CCD) detector. The measurement system converted the photon-counting head shown in Fig. 1 into the multichannel CCD detector. The measured temperatures in the region ranged from room temperature to $350^{\circ} \mathrm{C}$.

\subsection{Postannealing method}

The postannealing method is one of the TL glow curve component separation methods. This method utilizes the relationship between TL glow curves for a non-postannealed sample and a postannealed sample. The term postannealing here means to anneal at a setting temperature after irradiation. In the case of a TL glow curve that consists of one component, in spite of a decrease in TL peak intensity, the change does not occur at the TL glow peak temperature as a result of postannealing. A TL glow curve that consists of multiple components indicates that electrons in low trapping states are released by postannealing, whereas electrons in high trapping states are not. As a result, the TL glow peak temperature increases and the full width at half-maximum (FWHM) decreases. Figure 2 shows a theoretical TL glow curve using a general order that consists of two TL glow components. The TL parameters of Curve A are $E=1.44 \mathrm{eV}, s=10^{11} \mathrm{~s}^{-1}$, and $b=1.8$, and those for Curve B are $E=1.33 \mathrm{eV}, s=10^{11} \mathrm{~s}^{-1}$, and $b=1.8 . b$ is the probability rate of retrapping. The TL glow peak temperature is $200{ }^{\circ} \mathrm{C}$. In the case of postannealing at $120^{\circ} \mathrm{C}$, only the $\mathrm{TL}$ intensity of Curve B decreases. In addition, the TL glow peak temperature changes from 200 to $215^{\circ} \mathrm{C}$ and FWHM changes from 66 to $46^{\circ} \mathrm{C}$ due to the release of low trapping state electrons with high probability. The TL glow components can be discussed based on the changes in TL glow peak temperature, FWHM, and TL glow curve area with respect to the postannealing temperature.

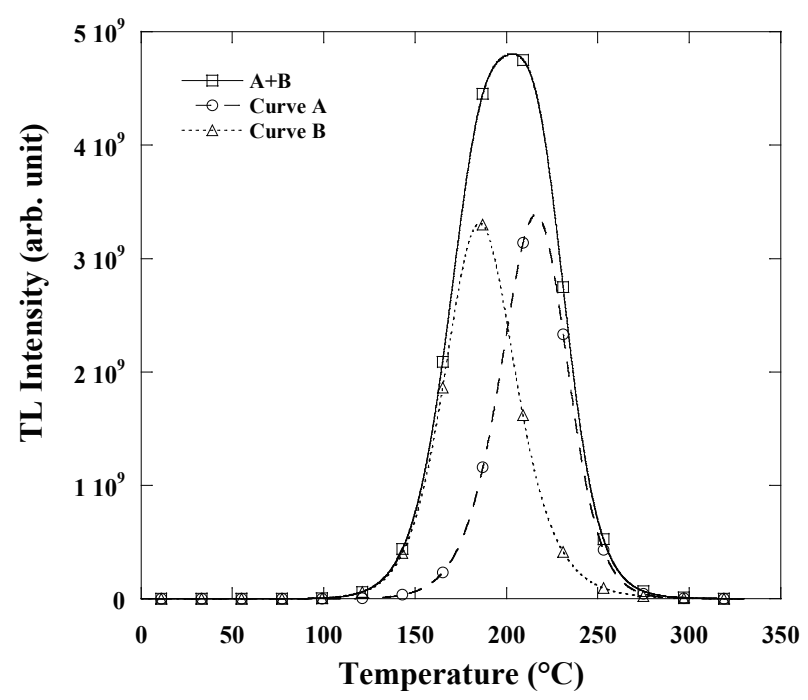

Fig. 2. Theoretical TL glow curve consisting of two glow components (Curve A: $E=1.44 \mathrm{eV}, s=10^{11} \mathrm{~s}^{-1}$, and $b=1.8$; Curve B: $E=1.33 \mathrm{eV}, s=10^{11} \mathrm{~s}^{-1}$, and $b=1.8$ ). 


\section{Results}

Figure 3 shows the TL glow curves obtained using the postannealing method. The TL glow peak temperatures for various postannealing temperatures are summarized in Table 1. The TL glow peak temperature for the non-postannealed sample was $160{ }^{\circ} \mathrm{C}$. The peak temperatures were 210 ${ }^{\circ} \mathrm{C}$ for the sample postannealed at 150 and $249{ }^{\circ} \mathrm{C}$ for the sample postannealed at $200{ }^{\circ} \mathrm{C}$. Thus, the TL glow curve temperature gradually shifted higher as the postannealing temperature increased. The FWHM also became gradually narrower, as shown in Fig. 3, which indicates that the TL glow peak temperature and FWHM of the TL glow curves for Na-doped BeO depend significantly on the postannealing temperature. Therefore, we can consider that the TL glow curves for Na-doped $\mathrm{BeO}$ consist of multiple components.

\section{Discussion}

Figure 3 shows that the TL glow curve for Na-doped $\mathrm{BeO}$ consists of multiple components. Here, we attempted to fit the TL glow curve using a general order equation for the sample postannealed at $200{ }^{\circ} \mathrm{C}$, because this TL glow curve had the least overlap of TL glow components. The general order kinetics indicates an intermediate between first-order and secondorder kinetics. ${ }^{(9)}$ Therefore, for the FWHM, first-order kinetics $<$ general-order kinetics $\leq$ secondorder kinetics. Even if the probability rate of retrapping $(b)$ changes, the TL glow peak temperature does not change, because it is determined by the trap depth $(E)$ and frequency factor $(s)$. In this

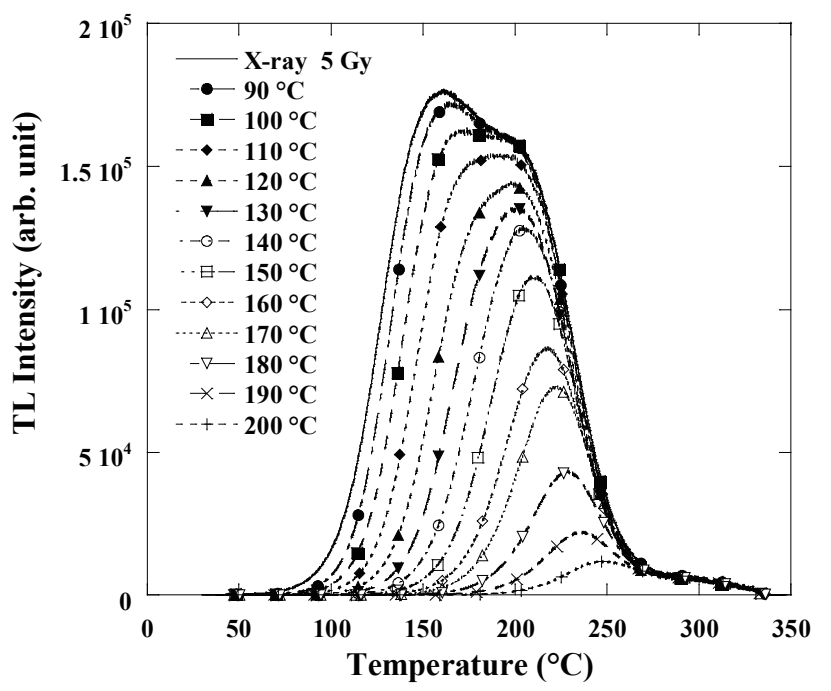

Fig. 3. TL glow curves for BeO:Na postannealed at various temperatures.

Table 1

TL glow peak temperatures for various postannealing temperatures.

\begin{tabular}{lccccccccccccc}
\hline $\begin{array}{l}\text { Postannealing } \\
\text { temperature }\left({ }^{\circ} \mathrm{C}\right)\end{array}$ & None & 90 & 100 & 110 & 120 & 130 & 140 & 150 & 160 & 170 & 180 & 190 & 200 \\
\hline $\begin{array}{l}\text { Glow peak } \\
\text { temperature }\left({ }^{\circ} \mathrm{C}\right)\end{array}$ & 161 & 164 & 173 & 190 & 198 & 198 & 205 & 210 & 217 & 223 & 228 & 231 & 249 \\
\hline
\end{tabular}


study, we considered that the general order provides the best fit for the TL glow curve shape of the sample postannealed at $200^{\circ} \mathrm{C}$. The equation for the general order fitting is given as

$$
I(T)=s n_{0} \exp \left(-\frac{E}{k T}\right)\left[1+\frac{s(b-1)}{\beta} \int_{T_{0}}^{T} \exp \left(-\frac{E}{k T}\right) d T^{\prime}\right]^{-\frac{b}{b-1}}
$$

where $I$ is the TL intensity, $n_{0}$ is the number of trapped electrons, $E$ is the trap depth $(\mathrm{eV}), k$ is the Boltzmann constant, $T$ is the absolute temperature $(\mathrm{K}), s$ is the frequency factor $\left(\mathrm{s}^{-1}\right)$, and $b$ is the probability rate of retrapping.

Figure 4(a) shows a TL glow curve for the sample postannealed at $200{ }^{\circ} \mathrm{C}$ measured at a heating rate of $0.1{ }^{\circ} \mathrm{C} \mathrm{s}^{-1}$ and the results of general-order fitting. The TL parameters were $E=$ $1.47 \mathrm{eV}, s=1 \times 10^{12} \mathrm{~s}^{-1}$, and $b=1.8$. Although a difference appeared at around $300{ }^{\circ} \mathrm{C}$ due to the presence of another TL glow component, the TL glow peak temperature and initial rise for the theoretical TL glow curve were in agreement with the experimental data. The accuracy of these TL parameters was then verified using the TL glow curve for a sample postannealed at $200{ }^{\circ} \mathrm{C}$ and measured at a heating rate of $0.01{ }^{\circ} \mathrm{C} \mathrm{s}^{-1}$ [Fig. 4(b)]. Despite the extremely low heating rate at $0.01{ }^{\circ} \mathrm{C} \mathrm{s}^{-1}$, the TL glow peak temperature and initial rise for the TL glow curve were in agreement with the experimental data. Slight differences in TL parameters cause large changes in TL glow peak temperature and FWHM; therefore, this result verified that the TL parameters used for the fitting were highly accurate. The glow component of around $300{ }^{\circ} \mathrm{C}$ has a lower signal noise ratio than other glow components due to heat radiation from a heater. Thus, we did not analyze the component.

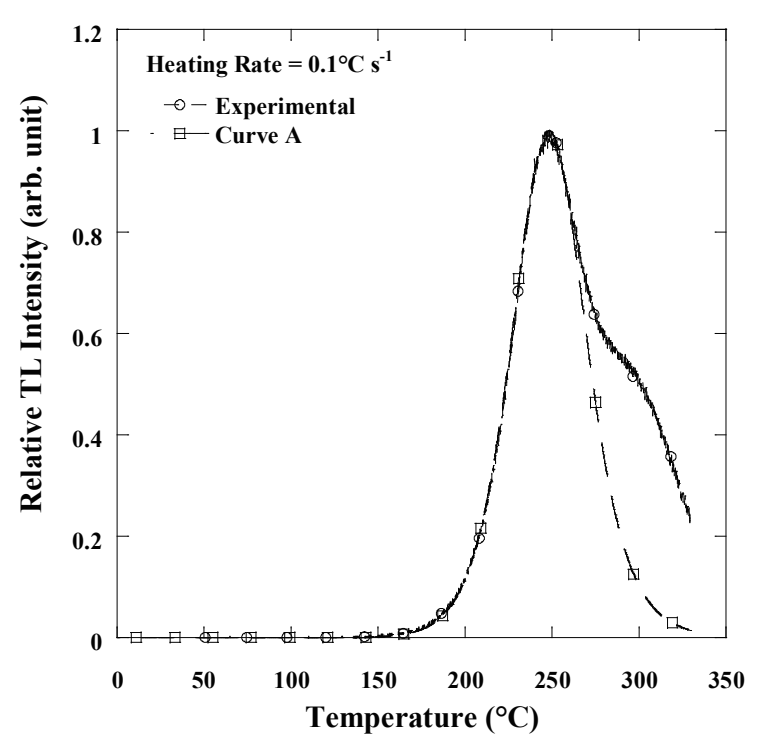

(a)

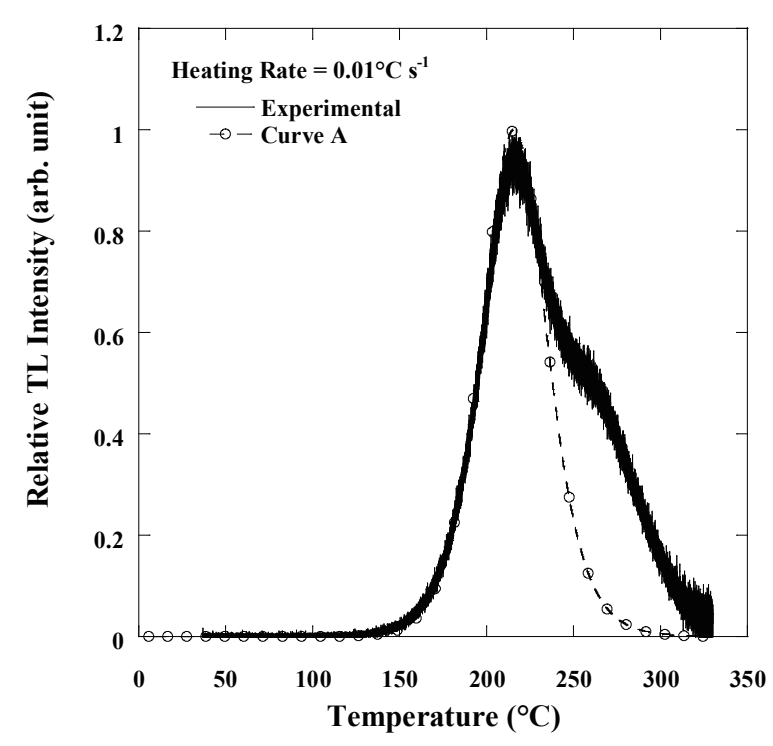

(b)

Fig. 4. (a) TL glow curve for the sample postannealed at $200{ }^{\circ} \mathrm{C}$ measured at a heating rate of $0.1{ }^{\circ} \mathrm{C} \mathrm{s}^{-1}$ and the general-order fitting $\left(E=1.47 \mathrm{eV}, s=1 \times 10^{12} \mathrm{~s}^{-1}\right.$, and $\left.b=1.8\right)$. (b) TL glow curve for the sample postannealed at $200{ }^{\circ} \mathrm{C}$ measured at a heating rate of $0.01{ }^{\circ} \mathrm{C} \mathrm{s}^{-1}$ and the general-order fitting $\left(E=1.47 \mathrm{eV}, s=1 \times 10^{12} \mathrm{~s}^{-1}\right.$, and $\left.b=1.8\right)$. 
Figure 5(a) shows the TL glow curve for a sample postannealed at $190{ }^{\circ} \mathrm{C}$, the theoretical TL glow curve $\left(E=1.47 \mathrm{eV}, s=1 \times 10^{12} \mathrm{~s}^{-1}\right.$, and $\left.b=1.8\right)$ for the sample postannealed at $200{ }^{\circ} \mathrm{C}$, and the difference between these TL glow curves. In Fig. 3, the TL glow peak temperatures for samples postannealed at 190 and $200{ }^{\circ} \mathrm{C}$ are 231 and $249{ }^{\circ} \mathrm{C}$, respectively. The difference in TL glow peak temperature indicates the presence of another TL glow component in Curve A $(E=1.47 \mathrm{eV}, s=1 \times$ $10^{12} \mathrm{~s}^{-1}$, and $\left.b=1.8\right)$ in this temperature region. The other TL glow components are made evident by the difference between the TL glow curves for postannealing at $190{ }^{\circ} \mathrm{C}$ and Curve A. The difference (Curve B) could then be fitted with TL parameters of $E=1.42 \mathrm{eV}, s=1 \times 10^{12} \mathrm{~s}^{-1}$, and $b=1.8$. The same frequency factor $(s)$ as that used for Curve A was used for two reasons. First, $s$ is generally dependent on the lattice vibration of the parent material. Second, the accuracy of the TL parameters for fitting Curve A for the TL glow curve of the sample postannealed at $200{ }^{\circ} \mathrm{C}$ is verified in Figs. 4(a) and 4(b). Figure 5(b) shows the TL glow curve for the sample postannealed at $190^{\circ} \mathrm{C}$, Curve A, Curve B, and the sum of Curves A and B. The TL glow peak temperature, initial rise, and FWHM for the sum of Curves A and B are in agreement with the experimental data for the sample postannealed at $190{ }^{\circ} \mathrm{C}$.

The results for samples postannealed in the range of $90-200{ }^{\circ} \mathrm{C}$ at $10{ }^{\circ} \mathrm{C}$ intervals were analyzed using the same method. The results of the component analysis are shown in Fig. 6 and suggest at least thirteen TL glow curve components. The TL parameters and TL glow peak temperatures for Na-doped $\mathrm{BeO}$ using the postannealing method at a very low heating rate are listed in Table 2. The TL parameters define $E=1.12-1.47 \mathrm{eV}, s=1 \times 10^{12} \mathrm{~s}^{-1}$, and $b=1.2-1.9$. These results were evaluated according to the figure of merit (FOM), which is defined as

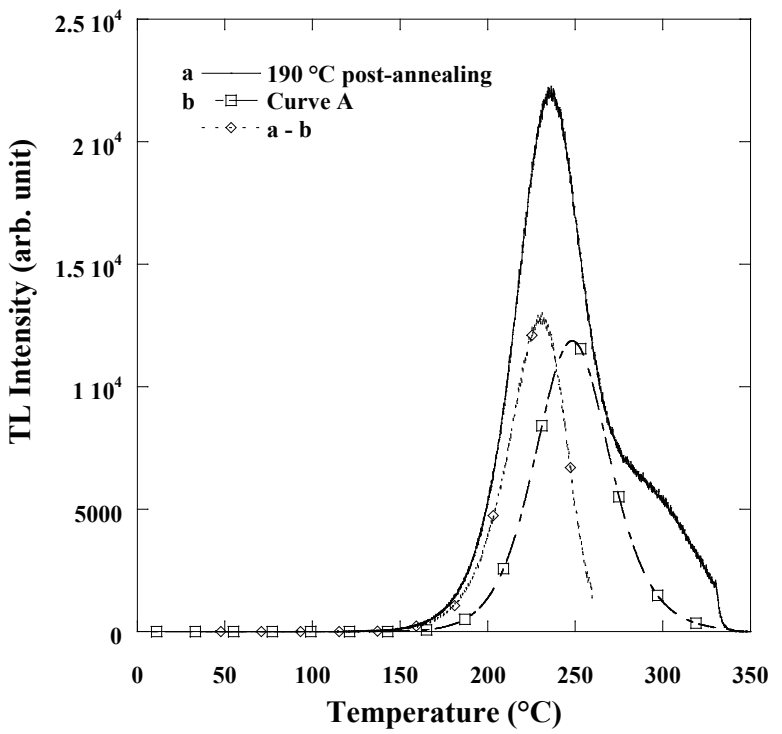

(a)

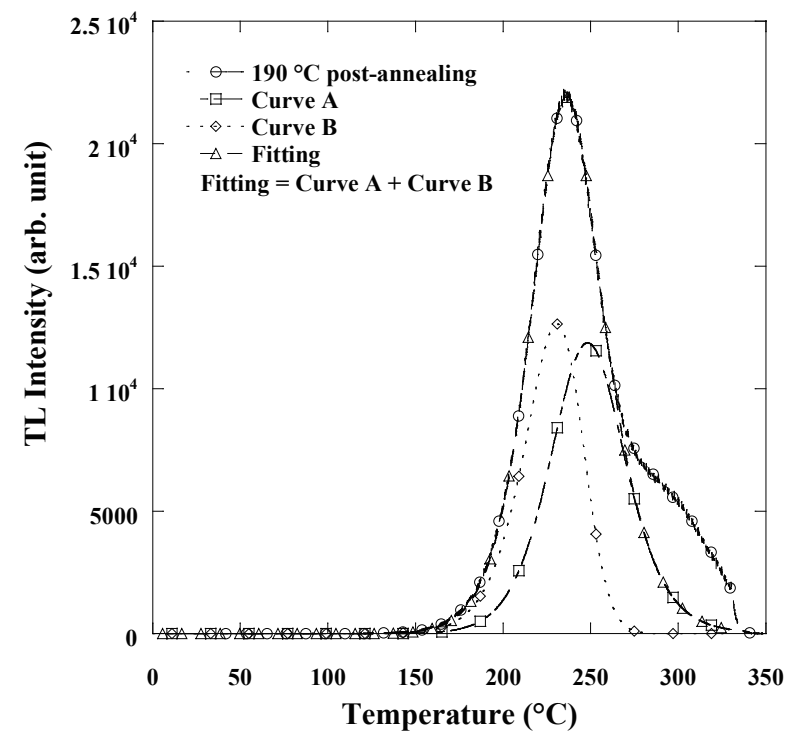

(b)

Fig. 5. (a) TL glow curve for a sample postannealed at $190{ }^{\circ} \mathrm{C}$, the theoretical glow curve (b: $E=1.47 \mathrm{eV}, s=1$ $\times 10^{12} \mathrm{~s}^{-1}$, and $b=1.8$ ) for the sample postannealed at $200^{\circ} \mathrm{C}$, and the difference between the glow curves $(\mathrm{a}-\mathrm{b})$. (b) Glow curve for the sample postannealed at $190{ }^{\circ} \mathrm{C}$, component Curve A, component Curve B, and the sum of Curves A and B. 


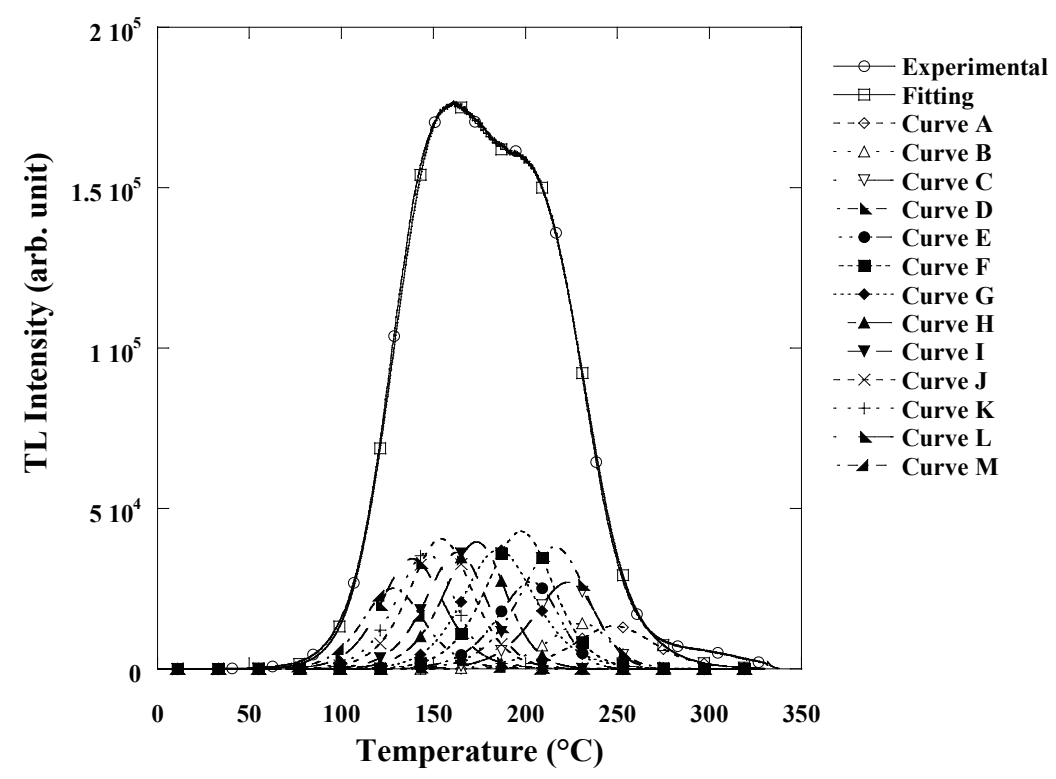

Fig. 6. TL glow curve analysis of thermoluminescent Na-doped $\mathrm{BeO}$ phosphor using the postannealing method at a very low heating rate.

Table 2

TL parameters and glow peak temperatures for BeO:Na using the postannealing method with a very low heating rate.

\begin{tabular}{lccccc}
\hline & $E(\mathrm{eV})$ & $s\left(\mathrm{~s}^{-1}\right)$ & $n_{0}$ & $b$ & $\begin{array}{c}\text { TL glow peak } \\
\text { temperature }\left({ }^{\circ} \mathrm{C}\right)\end{array}$ \\
\hline Peak A & 1.47 & $1 \times 10^{12}$ & $6.86 \times 10^{6}$ & 1.8 & 249 \\
Peak B & 1.42 & $1 \times 10^{12}$ & $5.62 \times 10^{6}$ & 1.2 & 231 \\
Peak C & 1.40 & $1 \times 10^{12}$ & $1.10 \times 10^{7}$ & 1.3 & 223 \\
Peak D & 1.37 & $1 \times 10^{12}$ & $1.65 \times 10^{7}$ & 1.5 & 216 \\
Peak E & 1.34 & $1 \times 10^{12}$ & $1.06 \times 10^{7}$ & 1.3 & 203 \\
Peak F & 1.32 & $1 \times 10^{12}$ & $1.86 \times 10^{7}$ & 1.6 & 198 \\
Peak G & 1.29 & $1 \times 10^{12}$ & $1.74 \times 10^{7}$ & 1.9 & 185 \\
Peak H & 1.25 & $1 \times 10^{12}$ & $1.57 \times 10^{7}$ & 1.5 & 174 \\
Peak I & 1.22 & $1 \times 10^{12}$ & $1.41 \times 10^{7}$ & 1.5 & 163 \\
Peak J & 1.20 & $1 \times 10^{12}$ & $1.66 \times 10^{7}$ & 1.7 & 154 \\
Peak K & 1.17 & $1 \times 10^{12}$ & $1.35 \times 10^{7}$ & 1.5 & 146 \\
Peak L & 1.15 & $1 \times 10^{12}$ & $1.40 \times 10^{7}$ & 1.8 & 139 \\
Peak M & 1.12 & $1 \times 10^{12}$ & $1.00 \times 10^{7}$ & 1.8 & 128 \\
\hline
\end{tabular}

$$
\operatorname{FOM}(\%)=\sum_{i}^{n} \frac{100\left|y_{i}-Y_{i}\right|}{A}
$$

where $n$ is the total number of channels, $i$ is the number of channels, $y_{i}$ is the measured TL intensity in channel- $i, Y_{i}$ is the fitting function in channel- $i$, and $A$ is the total TL integral of the fitted TL glow curve over all channels. ${ }^{(10)}$ The FOM determined from Fig. 6 was $0.38 \%$. The FOM for a TL glow curve analysis is typically around $3 \% ;^{(10)}$ therefore, the results indicate that the accuracy of the postannealing method at a very low heating rate was high. 


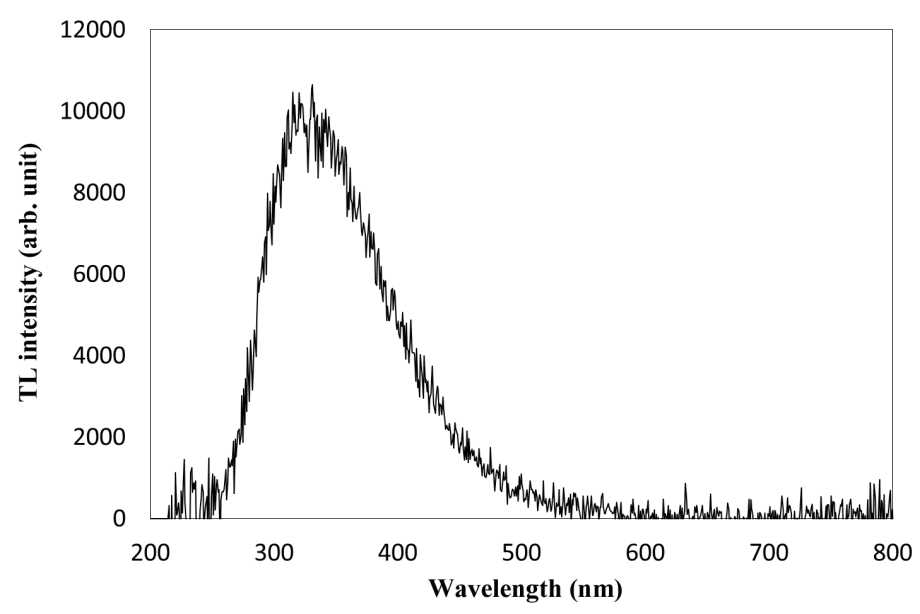

Fig. 7. TL spectra of thermoluminescent Na-doped $\mathrm{BeO}$ phosphor.

The crystal structure of $\mathrm{BeO}$ is characterized by the wurtzite structure. The cation site is at the center of a nearly perfect tetrahedron, with each beryllium atom surrounded by four oxygen atoms, and each oxygen atom by four beryllium atoms. There are seven $\mathrm{Be}-\mathrm{O}$ bonds and the axial bond is not equivalent to the basal bonds. As a result, there are two inequivalent Be sites. In Na-doped $\mathrm{BeO}, \mathrm{Na}^{+}$and other cation impurities substitute the Be sites. As a result, various kinds of vacancies are generated due to charge compensation. Furthermore, these vacancies function as electronic and hole traps by combining with anion vacancies, interstitial ions, and substitutional ions. Therefore, these considerations explain the $13 \mathrm{TL}$ glow components that we analyzed. Figure 7 shows the TL spectra of thermoluminescent Na-doped $\mathrm{BeO}$ phosphor. The TL wavelength was $325 \mathrm{~nm}$, and the emission was almost the same as the undoped $\mathrm{BeO}$ and Li-doped $\mathrm{BeO} \cdot{ }^{(1,11)}$ That is, $\mathrm{Na}^{+}$as a dopant has no role for TL emission.

The TL parameters for Na-doped $\mathrm{BeO}$ have not been reported until now. For undoped $\mathrm{BeO}$, Nieto et al. already reported the TL parameters obtained using an isothermal decay method. ${ }^{(7)}$ The main glow peak consisted of one component, and the TL parameters were $E=1.01 \mathrm{eV}$ and $s=1.8$ $\times 10^{9} \mathrm{~s}^{-1}$. The number of components of the main TL glow peak and the TL parameters differed in our analysis. In the future, we will try a TL glow curve analysis of thermoluminescent undoped $\mathrm{BeO}$ phosphor using a postannealing method with a very low heating rate to confirm the origin of the differences.

\section{Conclusion}

TL glow curves of the thermoluminescent Na-doped $\mathrm{BeO}$ phosphor were analyzed using a postannealing method at an extremely low heating rate of $0.1{ }^{\circ} \mathrm{C} \mathrm{s}^{-1}$. Although the TL glow curve for $\mathrm{Na}$-doped $\mathrm{BeO}$ was previously reported to consist only of a few components, this analysis indicated at least thirteen TL glow curve components. ${ }^{(2)}$ These TL parameters were $E=1.12-1.47$ $\mathrm{eV}, s=1 \times 10^{12}$, and $b=1.2-1.9$. The accuracy of the calculated TL parameters determined by the postannealing method at a very low heating rate was very high. When the results from this method were compared with those from electron spin resonance or infrared absorption spectroscopy, these results are expected to have an important role in the future for elucidating the TL mechanism. 


\section{Acknowledgement}

This work was partially supported by the Cooperative Research Project of Research Institute of Electronics, Shizuoka University.

\section{References}

1 E. Tochilin, N. Goldstein, and W. G. Miller: Health Phys. 16 (1969) 1.

2 Y. Yasuno, M. Ikendo, and T. Yamashita: National Tech. Rep. 21 (1975) 356.

3 S. Hisanaga, T. Yamashita, I. Kitamura, T. Itoh, and S. Kondo: Radioisotopes 39 (1990) 381.

4 C. Bacci, P. Bernardini, A. Damilano, C. Furetta, and B. Rispoli: J. Phys. D: Appl. Phys. 22 (1989) 1751.

5 D. R. Vij and N. Singh: J. Mater. Sci. 32 (1997) 2791.

6 B. Mukherjee: Radiat. Meas. 72 (2015) 31.

7 J. A. Nieto, C. A. Vega, T. R. Montalvo, and E. T. Cabrera: Appl. Radiat. Isot. 108 (2016) 8.

8 M. S. Rasheedy and E. M. Zahran: Phys. Scr. 73 (2006) 98.

9 C. E. May and J. A. Partridge: J. Chem. Phys. 40 (1964) 1401.

10 H. G. Balian and N. W. Eddy: Nucl. Instrum. Methods 145 (1977) 389.

11 Y. Yamashita, Y. Yasuno, and M. Ikedo: Health Phys. 27 (1974) 201. 